\title{
ASYMPTOTIC BEHAVIOUR OF THE SPECTRUM OF A DIRECT STRAIN FEEDBACK CONTROL SYSTEM
}

\author{
BAO ZHU GUO ${ }^{1}$
}

(Received 8 March 1993; revised 16 May 1994)

\begin{abstract}
This paper establishes an estimate for the asymptotic behaviour of the spectrum of a direct strain feedback (DSF) control system. The results show that the system operator corresponding to the closed loop system cannot have an analytic extension and that the decay rate for the system energy is not proportional to the feedback constant.
\end{abstract}

\section{Introduction}

Direct strain feedback (DSF) is used in practise to control the vibration of flexible arms. It is known for its effectiveness and simplicity of implementation in many applications (see [2]). However, no rigorous theoretical results were available until the work of [3]. In [3], it was shown that the closed loop DSF control system is asymptotically stable. In fact, the DSF can make the system exponentially stable because it is equivalent to the standard one-end stabilizer system which has been studied extensively in [1] and [4]. In this paper, we shall establish an estimate for the asymptotic behaviour of the spectrum for a typical DSF control system. The results show that the system operator corresponding to the closed loop system cannot have an analytic extension and that the decay rate for the system energy is not proportional to the feedback constant. These results may help us to understand qualitatively the effect of DSF in the control of the vibration of flexible robot arms.

Suppose there is a single-link uniform flexible arm of length $\ell$. One end of the arm is attached to the shaft of a control motor which rotates it in the horizontal plane. Let $y(x, t)$ denote the bending displacement of the arm at time $t$ and at a distance $x$ from the base. Then, if the other end of the flexible arm is free, the dynamics of the closed loop system of bending vibration of the flexible arms with DSF control are known

'Institute of Systems Science, Academia Sinica, Beijing 100080, China.

(C) Australian Mathematical Society, 1995, Serial-fee code 0334-2700/95 
(see [3]):

$$
\left\{\begin{array}{l}
\frac{\partial^{2} y(x, t)}{\partial t^{2}}+\frac{\partial^{4} y(x, t)}{\partial t^{4}}+k x \frac{\partial^{3} y(0, t)}{\partial t \partial x^{2}}=0, \\
y(0, t)=y^{\prime}(0, t)=y^{\prime \prime}(\ell, t)=y^{\prime \prime \prime}(\ell, t)=0,
\end{array} \quad 0<x<\ell,\right.
$$

where $k>0$ is the feedback constant. To simplify notation, the ratio of bending rigidity with the mass density is taken to be 1 . Define the operators $\mathbb{A}$ and $\mathbb{B}$ in $\mathscr{H}=L^{2}(0, \ell)$ as follows:

$$
\left\{\begin{aligned}
\mathbb{A} \phi(x) & =\phi^{\prime \prime \prime \prime}(x), \\
D(\mathbb{A}) & =\left\{\phi(x) \in \mathscr{H} \mid \phi(0)=\phi^{\prime}(0)=\phi^{\prime \prime}(\ell)=\phi^{\prime \prime \prime}(\ell)=0\right\}, \\
\mathbb{B} \phi(x) & =x \phi^{\prime \prime}(0) .
\end{aligned}\right.
$$

Then (1) can be written as the following second order evolution equation in $\mathscr{H}$ :

$$
y_{t t}(t, x)+\mathbb{A} y(t, x)+k \mathbb{B} y_{t}(t, x)=0 .
$$

The following results are known (see $[1,4]$ ).

THEOREM 1. The system (3) admits a unique solution in $\mathscr{H} \times \mathscr{H}$

$$
\left[\begin{array}{c}
\mathbb{A} y \\
\mathbb{A}^{\frac{1}{2}} y_{t}
\end{array}\right]=e^{\mathscr{A} t}\left[\begin{array}{c}
\mathbb{A} y_{0} \\
\mathbb{A}^{\frac{1}{2}} y_{1}
\end{array}\right]
$$

for any initial condition $\left(y(x, 0), y_{t}(x, 0)\right)=\left(y_{0}(x), y_{1}(x)\right) \in D(\mathbb{A}) \times D\left(\mathbb{A}^{\frac{1}{2}}\right)$. Fur thermore, it satisfies the following uniform exponential decay

$$
\left\|e^{\mathscr{A} t}\right\| \leq M e^{-\omega t} \text { for some } M>1 \text { and } \omega>0,
$$

where $e^{d t}$ is the $C_{0}$-semigroup generated by the system operator

$$
\mathscr{A}=\left[\begin{array}{cc}
0 & \mathbb{A}^{\frac{1}{2}} \\
-\mathbb{A}^{\frac{1}{2}} & 0
\end{array}\right]\left[\begin{array}{cc}
0 & I \\
I & k B A^{-\frac{1}{2}}
\end{array}\right] .
$$

Theorem 1 is significant, as it indicates that the DSF can make the system exponentially stable.

\section{Spectral analysis}

We shall characterize the spectrum of the operator $\mathscr{A}$ in the sequel. The following well-known results about the operator $\mathscr{A}$ are used frequently throughout the paper. 
LEMMA 1. (i) $\mathbb{A}$ is self-adjoint and positive definite on $\mathscr{H}$ and has eigen elements $\left\{\left(\lambda_{n}, \phi_{n}\right)\right\}_{n=1}^{\infty}$

(ii) $\lambda_{n}=\beta_{n}^{4}>0, \quad \beta_{n}=o(n), \quad 2+\left[\exp \left(\beta_{n} l\right)+\exp \left(-\beta_{n} \ell\right)\right] \cos \left(\beta_{n} \ell\right)=0$;

(iii) $\left\{\phi_{n}\right\}$ forms an orthogonal basis of $\mathscr{H}$ and can be expressed as:

$$
\left\{\begin{array}{l}
\phi_{n}(x)=-\frac{1+\gamma}{2} \exp \left(\beta_{n} x\right)-\frac{1-\gamma}{2} \exp \left(-\beta_{n} x\right)+\gamma \sin \left(\beta_{n} x\right)+\cos \left(\beta_{n} x\right), \\
\gamma=-\frac{\exp \left(\beta_{n} \ell\right)-\sin \left(\beta_{n} \ell\right)+\cos \left(\beta_{n} \ell\right)}{\exp \left(\beta_{n} \ell\right)+\sin \left(\beta_{n} \ell\right)+\cos \left(\beta_{n} \ell\right)} \rightarrow-1 \quad \text { as } n \rightarrow \infty .
\end{array}\right.
$$

(iv) Let

$$
x=\sum_{n=1}^{\infty} b_{n} \phi_{n}(x) \quad \text { in } \mathscr{H} .
$$

Then

$$
\phi_{n}^{\prime \prime}(0)=-2 \beta_{n}^{2}, \quad b_{n}=o\left(\beta_{n}^{-2}\right), \quad\left\|\phi_{n}\right\|=o(1) .
$$

Since $\sigma(\mathscr{A})$ consists of only eigenvalues of operator $\mathscr{A}$, it is easy to show that a necessary and sufficient condition for $\lambda \in \sigma(\mathscr{A})$ is that there exists a nonzero element $\phi \neq 0$ satisfying the second order eigenvalue problem:

$$
\left\{\begin{array}{l}
\lambda^{2} \phi(x)+\phi^{\prime \prime \prime \prime}(x)+k x \lambda \phi^{\prime \prime}(0)=0, \\
\phi(0)=\phi^{\prime}(0)=\phi^{\prime \prime}(\ell)=\phi^{\prime \prime \prime}(\ell)=0
\end{array}\right.
$$

or

$$
\lambda^{2} \phi+\mathbb{A} \phi+k \lambda \mathbb{B} \phi=0,
$$

in operator form. By Theorem 1,

$$
\operatorname{Re} \lambda \leq-\omega<0 .
$$

From Lemma 1 and (7), if $(\lambda, \phi), \phi \neq 0$, is a solution of (6), then $\lambda^{2} \in \rho(-\mathbb{A})$, the resolvent set of the operator $-\mathbb{A}$. Therefore

$$
\phi=-k \lambda \phi^{\prime \prime}(0)\left(\lambda^{2}+A\right)^{-1} x .
$$

On the other hand, by twice integrating (5) from $\ell$ to $x$, we obtain

$$
\phi^{\prime \prime}(0)\left(1+k \lambda \frac{\ell^{3}}{3}\right)+\lambda^{2} \int_{0}^{\ell} x \phi(x) d x=0 .
$$

Substituting (9) into (8), we have

$$
\begin{aligned}
& \phi^{\prime \prime}(0) {\left[1+k \lambda \frac{\ell^{3}}{3}-k \lambda^{3} \int_{0}^{\ell} x\left[\left(\lambda^{2}+\mathbb{A}\right)^{-1} x\right] d x\right] } \\
&=\phi^{\prime \prime}(0)\left[1+k \lambda \int_{0}^{\ell} x\left[\mathbb{A}\left(\lambda^{2}+\mathbb{A}\right)^{-1} x\right] d x\right]=0 .
\end{aligned}
$$


So $\phi \neq 0$ if and only if $1+k \lambda \int_{0}^{\ell} x\left[\mathbb{A}\left(\lambda^{2}+\mathbb{A}\right)^{-1} x\right] d x=0$. Thus we have proved the following:

LEMMA 2. A necessary and sufficient condition for $(\lambda, \phi), \phi \neq 0$, to be a solution of (5) is that

$$
\begin{aligned}
F(\lambda) & =1+k \lambda \int_{0}^{\ell} x\left[\mathbb{A}\left(\lambda^{2}+\mathbb{A}\right)^{-1} x\right] d x \\
& =1+k \lambda \sum_{n=1}^{\infty} \frac{\lambda_{n}}{\lambda^{2}+\lambda_{n}} b_{n}^{2}\left\|\phi_{n}\right\|^{2}=0,
\end{aligned}
$$

that is, $\lambda$ is a zero point of the meromorphic function $F(\lambda)$. Meanwhile

$$
\phi(x)=\left(\lambda^{2}+\mathbb{A}\right)^{-1} x
$$

LEMMA 3. Let $F(\lambda)$ and $b_{n}, n \geq 1$ be defined as in (10). Then $b_{n} \neq 0$ for every $n \geq 1$, and

$$
F(\lambda)=1+\frac{k}{\ell} \frac{f^{\prime}(\sqrt{2 \lambda})}{f(\sqrt{2 \lambda})},
$$

where

$$
f(\lambda)=4+\exp (i \lambda \ell)+\exp (-i \lambda \ell)+\exp (\lambda \ell)+\exp (-\lambda \ell),
$$

that is, $F(\lambda)=0$ if and only if $f(\sqrt{2 \lambda})+\frac{k}{\ell} f^{\prime}(\sqrt{2 \lambda})=0, \quad\left\{ \pm i \beta_{n}^{2}\right\}=\left\{ \pm i \sqrt{\lambda_{n}}\right\}$ consists of the poles of the function $F(\lambda)$.

PROOF. Let $y(x)=\left(\lambda^{2}+\mathbb{A}\right)^{-1} x$. Then

$$
\left\{\begin{array}{l}
\lambda^{2} y(x)+y^{\prime \prime \prime \prime}(x)=x \\
y(0)=y^{\prime}(0)=y^{\prime \prime}(\ell)=y^{\prime \prime \prime}(\ell)=0
\end{array}\right.
$$

Taking the inner product with $x$ on both sides of the above equation, we obtain

$$
\lambda^{2} \int_{0}^{\ell} x y(x) d x+y^{\prime \prime}(0)=\frac{\ell^{3}}{3} \text {. }
$$

Therefore

$$
\begin{aligned}
F(\lambda) & =1+k \lambda \frac{\ell^{3}}{3}-k \lambda^{3} \int_{0}^{\ell} x y(x) d x \\
& =1+k \lambda y^{\prime \prime}(0)=1+k \frac{g^{\prime \prime}(0)}{\lambda},
\end{aligned}
$$

where $g(x)$ is the solution of

$$
\left\{\begin{array}{l}
\lambda^{2} g(x)+g^{\prime \prime \prime \prime}(x)=0, \\
g(0)=g^{\prime \prime}(\ell)=g^{\prime \prime \prime}(\ell)=0, \quad g^{\prime}(0)=-1
\end{array}\right.
$$


Throughout this paper, we use $\sqrt{\lambda}$ to denote the positive branch of the complex function $\lambda$, that is,

$$
\left\{\begin{array}{l}
\lambda=\rho e^{i \theta}, \quad-\pi<\vartheta \leq \pi \\
\sqrt{\lambda}=\sqrt{\rho} e^{\frac{i 0}{2}}, \quad \sqrt{\lambda i}=e^{\frac{i \pi}{4}} \sqrt{\lambda}, \quad \sqrt{2 \lambda}=\sqrt{2} \cdot \sqrt{\lambda} .
\end{array}\right.
$$

Now the solution of (14) can be written as

$$
g(x)=a_{1} \exp (\sqrt{\lambda i} x)+a_{2} \exp (-\sqrt{\lambda i} x)+a_{3} \sin (\sqrt{\lambda i} x)+a_{4} \cos (\sqrt{\lambda i} x),
$$

where $a_{i}, i=1,2,3,4$, are determined by the boundary conditions of (14), that is,

$\left[\begin{array}{cccc}1 & 1 & 0 & 1 \\ 1 & -1 & -1 & 0 \\ \exp (\sqrt{\lambda i} \ell) & \exp (-\sqrt{\lambda i} \ell) & -\sin (\sqrt{\lambda i} \ell) & -\cos (\sqrt{\lambda i} \ell) \\ \exp (\sqrt{\lambda i} \ell) & -\exp (-\sqrt{\lambda i} \ell) & -\cos (\sqrt{\lambda i} \ell) & \sin (\sqrt{\lambda i} \ell)\end{array}\right]\left[\begin{array}{c}a_{1} \\ a_{2} \\ a_{3} \\ a_{4}\end{array}\right]=\left[\begin{array}{c}0 \\ -\frac{1}{\sqrt{\lambda i}} \\ 0 \\ 0\end{array}\right]$.

Solving this algebraic equation, we obtain

$a_{4}=\frac{1}{2 \sqrt{\lambda i}} \frac{\sin (\sqrt{\lambda i} \ell)[\exp (\sqrt{\lambda i} \ell)+\exp (-\sqrt{\lambda i} \ell)]-\cos (\sqrt{\lambda i} \ell)[\exp (\sqrt{\lambda i} \ell)-\exp (-\sqrt{\lambda i} \ell)]}{2+\cos (\sqrt{\lambda i} \ell)[\exp (\sqrt{\lambda i} \ell)+\exp (-\sqrt{\lambda i} \ell)]}$.

We observe that

$$
\left\{\begin{array}{l}
\sin (\sqrt{\lambda i} \ell)=[\exp (i \sqrt{\lambda i} \ell)-\exp (-i \sqrt{\lambda i} \ell)] / 2 i \\
\cos (\sqrt{\lambda i} \ell)=[\exp (i \sqrt{\lambda i} \ell)+\exp (-i \sqrt{\lambda i} \ell)] / 2 \\
\frac{i+1}{\sqrt{i}}=\sqrt{2}, \frac{1-i}{\sqrt{i}}=-\sqrt{2} i,(1+i) \sqrt{\lambda i}=i \sqrt{2 \lambda},(1-i) \sqrt{\lambda i}=\sqrt{2 \lambda}
\end{array}\right.
$$

Thus

$$
a_{4}=\frac{1}{\sqrt{2 \lambda}} \frac{-[\exp (i \sqrt{2 \lambda} \ell)-\exp (-i \sqrt{2 \lambda} \ell)]+i[\exp (\sqrt{2 \lambda} \ell)-\exp (-\sqrt{2 \lambda} \ell)]}{4+\exp (i \sqrt{2 \lambda} \ell)+\exp (-i \sqrt{2 \lambda} \ell)+\exp (\sqrt{2 \lambda} \ell)+\exp (-\sqrt{2 \lambda} \ell)]} .
$$

Finally, we obtain the explicit expression of $F(\lambda)$ :

$$
\begin{aligned}
F(\lambda) & =1+k \frac{g^{\prime \prime}(0)}{\lambda}=1+\frac{k}{\lambda}\left(-2 a_{4} \lambda i\right)=1-2 k a_{4} i \\
& =1+k \frac{2}{\sqrt{2 \lambda}} \frac{i[\exp (i \sqrt{2 \lambda} \ell)-\exp (-i \sqrt{2 \lambda} \ell)]+\exp (\sqrt{2 \lambda} \ell)-\exp (-\sqrt{2 \lambda} \ell)}{4 \sqrt{2 \lambda} \ell)+\exp (-i \sqrt{2 \lambda} \ell)+\exp (\sqrt{2 \lambda} \ell)+\exp (-\sqrt{2 \lambda} \ell)} \\
& =1+\frac{k}{\ell} \frac{f^{\prime}(\sqrt{2 \lambda})}{f(\sqrt{2 \lambda})} .
\end{aligned}
$$


which is (12). Using a Taylor's expansion, both $f(\sqrt{2 \lambda})$ and $f^{\prime}(\sqrt{2 \lambda})$ are entire functions of $\lambda$. Let $\sqrt{2 \lambda}=a+i b$. Then it follows that

$$
\begin{aligned}
f(\sqrt{2 \lambda})=4 & +\left(e^{b \ell}+e^{-b \ell}\right) \cos (a \ell)+\left(e^{a \ell}+e^{-a \ell}\right) \cos (b \ell) \\
& +i\left[\left(e^{-b \ell}-e^{b \ell}\right) \sin (a \ell)+\left(e^{a \ell}-e^{-a \ell}\right) \sin (b \ell)\right], \\
\frac{\sqrt{2 \lambda}}{2} f^{\prime}(\sqrt{2 \lambda})= & -\left(e^{b \ell}+e^{-b \ell}\right) \sin (a \ell)+\left(e^{a \ell}-e^{-a \ell}\right) \cos (b \ell) \\
& +i\left[\left(e^{-b \ell}-e^{b \ell}\right) \cos (a \ell)+\left(e^{a \ell}+e^{-a \ell}\right) \sin (b \ell)\right] .
\end{aligned}
$$

By (ii) of Lemma 1, it can be easily shown that

$$
f\left(\sqrt{2\left( \pm i \beta_{n}^{2}\right)}\right)=4+2\left[\exp \left(\beta_{n} \ell\right)+\exp \left(\beta_{n} \ell\right)\right] \cos \left(\beta_{n} \ell\right)=0
$$

and by (10), we see that $\left\{ \pm i \beta_{n}^{2}\right\}$ consists of all zeros of $f(\lambda)$. Also $\left\{ \pm i \beta_{n}^{2}\right\}$ are the poles of $F(\lambda)$. In fact, if $f^{\prime}\left(\sqrt{2\left( \pm i \beta_{n}^{2}\right)}\right)=0$, then

$$
\begin{aligned}
& -\left[\exp \left(\beta_{n} \ell\right)+\exp \left(-\beta_{n} \ell\right)\right] \sin \left(\beta_{n} \ell\right)+\left[\exp \left(\beta_{n} \ell\right)-\exp \left(-\beta_{n} \ell\right)\right] \cos \left(\beta_{n} \ell\right) \\
& +i\left[\left[\exp \left(\beta_{n} \ell\right)+\exp \left(-\beta_{n} \ell\right)\right] \sin \left(\beta_{n} \ell\right)-\left[\exp \left(\beta_{n} \ell\right)-\exp \left(-\beta_{n} \ell\right)\right] \cos \left(\beta_{n} \ell\right)\right]=0
\end{aligned}
$$

and hence

$$
\left[\exp \left(\beta_{n} \ell\right)+\exp \left(-\beta_{n} \ell\right)\right] \sin \left(\beta_{n} \ell\right)=\left[\exp \left(\beta_{n} \ell\right)-\exp \left(-\beta_{n} \ell\right)\right] \cos \left(\beta_{n} \ell\right)
$$

or

$$
\sin \left(\beta_{n} \ell\right)=-2\left[\exp \left(\beta_{n} \ell\right)-\exp \left(-\beta_{n} \ell\right)\right] /\left[\exp \left(\beta_{n} \ell\right)+\exp \left(-\beta_{n} \ell\right)\right]^{2},
$$

by (ii) of Lemma 1 . Since

$$
\cos \left(\beta_{n} \ell\right)=-2 /\left[\exp \left(\beta_{n} \ell\right)+\exp \left(-\beta_{n} \ell\right)\right]
$$

we have

$$
\sin ^{2}\left(\beta_{n} \ell\right)+\cos ^{2}\left(\beta_{n} \ell\right)=\left\{2 /\left[\exp \left(\beta_{n} \ell\right)+\exp \left(-\beta_{n} \ell\right)\right]\right\}^{2}<1,
$$

which is a contradiction. Thus

$$
|f(\lambda)|+\left|f^{\prime}(\lambda)\right| \neq 0
$$

and the proof is complete.

In view of (15), the analytical transformation $\sqrt{2 \lambda}$ maps the positive imaginary axis onto a ray $\gamma=\{\lambda \mid \operatorname{Re} \lambda=\operatorname{Im} \lambda\}$ of the first quadrant and maps the negative imaginary axis onto a ray $\bar{\gamma}=\{\lambda \mid \operatorname{Re} \lambda=-\operatorname{Im} \lambda\}$ of the fourth quadrant. Meanwhile, $\beta_{n}^{2}$ corresponds to $\hat{\lambda}_{n}=e^{\frac{i \pi}{4}} \beta_{n}$ on $\gamma$ and $-\beta_{n}^{2}$ to $\hat{\lambda}_{n}=e^{\frac{i \pi}{4}} \beta_{n}$, respectively.

Our main result is the following. 
THEOREM 2. There is an infinite number of eigenvalues $\left\{\mu_{n}, \bar{\mu}_{n}\right\}_{1}^{\infty}$ to (6). Furthermore, they posses the following asymptotic property:

$$
\lim _{n \rightarrow \infty} d\left(\mu_{n}, \hat{O}_{n}\right)=0 \text {, }
$$

where $\bar{\mu}_{n}$ is the conjecture of $\mu_{n}$, and $\hat{O}_{n}$ is the circle with center at $i \sqrt{\lambda_{n}}$ and radius $2 k / \ell$.

PROOF. From (12) and the previous discussion, let us investigate the values of $2 f^{\prime}(\lambda) /(\lambda f(\lambda))$ near $\hat{\lambda}_{n}$ and $\overline{\hat{\lambda}}_{n}$. By the conjugation relation

$$
\overline{2 f^{\prime}(\lambda) /(\lambda f(\lambda))}=2 f^{\prime}(\bar{\lambda}) /(\bar{\lambda} f(\bar{\lambda})),
$$

it suffices to consider only such $\lambda$ located in the first quadrant (see Figure 1 below). Let $\lambda=a+b i$. Then

$$
\begin{aligned}
f(\lambda)= & 4+\left(e^{b \ell}+e^{-b \ell}\right) \cos (a \ell)+\left(e^{a \ell}+e^{-a \ell}\right) \cos (b \ell) \\
& +i\left[\left(e^{-b \ell}-e^{b \ell}\right) \sin (a \ell)+\left(e^{a \ell}-e^{-a \ell}\right) \sin (b \ell)\right] \\
f^{\prime}(\lambda)= & -\left(e^{b \ell}+e^{-b \ell}\right) \sin (a \ell)+\left(e^{a \ell}-e^{-a \ell}\right) \cos (b \ell) \\
& +i\left[\left(e^{-b \ell}-e^{b \ell}\right) \cos (a \ell)+\left(e^{a \ell}+e^{-a \ell}\right) \sin (b \ell)\right] .
\end{aligned}
$$

Since $f(\lambda)$ is symmetric about $a$ and $b$, it is sufficient to consider only the case of $b \geq a$.

Now for a sufficiently small $\rho>0$ and for every integer $n$, take $\mathscr{O}_{n}$ to be the circle with center at $\hat{\lambda}_{n}$ and radius $\rho$ such that $\mathscr{O}_{n} \cap \mathscr{O}_{m}=\emptyset$ if $n \neq m$ (Figure 1). This is possible because $\hat{\lambda}_{n}=o(n)$.

Let $\lambda=a+b \in \Gamma_{n}(b \geq a)$, the circumference of circle $\mathscr{O}_{n}$, that is,

$$
a=\beta_{n}+\rho \cos \theta, \quad b=\beta_{n}+\rho \sin \theta, \quad \pi / 4 \leq \theta \leq \pi / 4+\pi .
$$

Then

$$
\begin{aligned}
e^{b \ell} f(\lambda) & =4 e^{-b \ell}+\left(1+e^{-2 b \ell}\right) \cos (a \ell)+\left(e^{(a-b) \ell}+e^{-(a+b) \ell)}\right) \cos (b \ell) \\
& +i\left[\left(e^{-2 b \ell}-1\right) \sin (a \ell)+\left(e^{(a-b) \ell}-e^{-(a+b) \ell}\right) \sin (b \ell)\right] \\
& =\cos (a \ell)+e^{(a-b) \ell} \cos (b \ell)+i\left[-\sin (a \ell)+e^{(a-b) \ell} \sin (b \ell)\right] \\
& +e^{-b \ell}\left[4+e^{-b \ell} \cos (a \ell)+e^{-a \ell} \cos (b \ell)+i\left[\left(e^{-b \ell} \sin (a \ell)-e^{-a \ell} \sin (b \ell)\right]\right],\right. \\
e^{b \ell} f^{\prime}(\lambda) & =-\sin (a \ell)+e^{(a-b) \ell} \cos (b \ell)+i\left[-\cos (a \ell)+e^{(a-b) \ell} \sin (b \ell)\right] \\
& +e^{-b \ell}\left[-e^{-b \ell} \sin (a \ell)-e^{-a \ell} \cos (b \ell)+i\left[\left(e^{-b \ell} \cos (a \ell)+e^{-a \ell} \sin (b \ell)\right]\right]\right.
\end{aligned}
$$




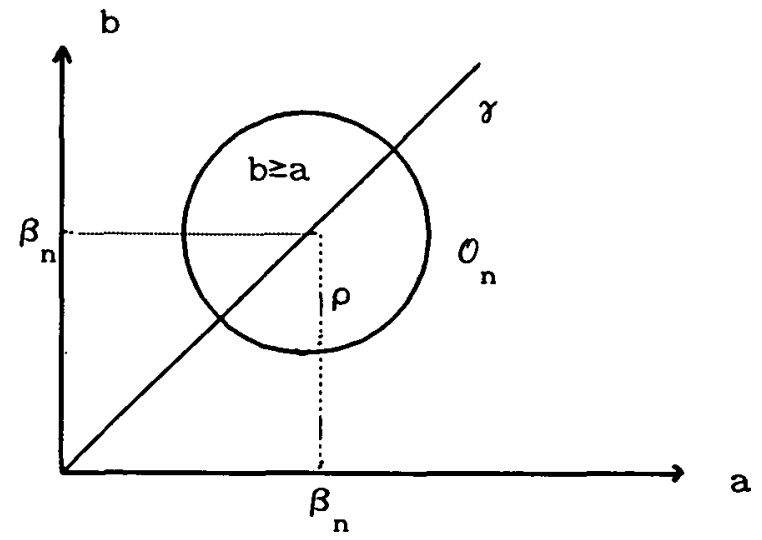

FIGURE 1. The region enclosed by circles with center at $\lambda_{n}$ and radius $\rho$.

and hence

$$
\left\{\begin{array}{l}
\left|\frac{f^{\prime}(\lambda)}{f(\lambda)}\right|^{2} \leq \frac{1+\exp (2(a-b) \ell)-2 \exp ((a-b) \ell) \sin ((a+b) \ell)+48 e^{-b \ell}}{1+\exp (2(a-b) \ell)+2 \exp ((a-b) \ell) \cos ((a+b) \ell)+128 e^{-b \ell}} \\
\left|\frac{f^{\prime}(\lambda)}{f(\lambda)}\right|^{2} \geq \frac{1+\exp (2(a-b) \ell)-2 \exp ((a-b) \ell) \sin ((a+b) \ell)-48 e^{-b \ell}}{1+\exp (2(a-b) \ell)+2 \exp ((a-b) \ell) \cos ((a+b) \ell)+128 e^{-b \ell}}
\end{array}\right.
$$

Furthermore,

$$
\begin{aligned}
1+\exp (2(a- & b) \ell)+2 \exp ((a-b) \ell) \cos ((a+b) \ell)-128 e^{-b \ell} \\
\geq & {[1-\exp (\rho(\cos \theta-\sin \theta) \ell)]^{2} } \\
+ & 2 \exp (\rho(\cos \theta-\sin \theta) \ell)[1-\cos (\rho(\cos \theta+\sin \theta) \ell)] \\
+ & 2 \exp (\rho(\cos \theta-\sin \theta) \ell)\left[\left[\cos \left(2 \beta_{n} \ell\right)+1\right] \cos (\rho(\cos \theta+\sin \theta) \ell)\right. \\
& -\sin \left(2 \beta_{n} \ell\right) \sin (\rho(\sin \theta+\sin \theta) \ell]-128 e^{\rho \ell} e^{-\beta_{n} \ell} \\
\rightarrow & {[1-\exp (\rho(\cos \theta-\sin \theta) \ell)]^{2} } \\
& +2 \exp (\rho(\cos \theta-\sin \theta) \ell)[1-\cos (\rho(\cos \theta+\sin \theta) \ell)]
\end{aligned}
$$

as $n \rightarrow \infty$ uniformly for all $\lambda \in \Gamma_{n}$. Here we have used the fact that

$$
\left\{\begin{array}{l}
\cos \left(\beta_{n} \ell\right)=\frac{2}{\exp \left(\beta_{n} \ell\right)+\exp \left(-\beta_{n} \ell\right)}=o\left(\exp \left(-\beta_{n} \ell\right)\right) \\
1+\cos \left(2 \beta_{n} \ell\right)=2 \cos ^{2}\left(\beta_{n} \ell\right)=o\left(\exp \left(-\beta_{n} \ell\right)\right) \\
\sin \left(2 \beta_{n} \ell\right)=2 \sin \left(\beta_{n} \ell\right) \cos \left(\beta_{n} \ell\right)=o\left(\exp \left(-\beta_{n} \ell\right)\right)
\end{array}\right.
$$

Let

$$
\begin{aligned}
c(\rho, \theta) & =[1-\exp (\rho(\cos \theta-\sin \theta) \ell)]^{2} \\
& +2 \exp (\rho(\cos \theta-\sin \theta) \ell)[1-\cos (\rho(\cos \theta+\sin \theta) \ell)]
\end{aligned}
$$


Then, $c(\rho, \theta) \geq 0$ for any $\rho>0$, and $\theta \in[\pi / 4, \pi / 4+\pi]$. If $c(\rho, \theta)=0$, then

$$
\cos \theta-\sin \theta=\cos \theta+\sin \theta=0,
$$

which leads to a contradiction for the case of $2 \rho \ell<2 \pi$. Hence $c_{0}(\rho)=\min c^{\frac{1}{2}}(\rho, \theta)>0$. Finally we obtain

$$
\begin{aligned}
& \left|\frac{2}{\lambda} \frac{f^{\prime}(\lambda)}{f(\lambda)}\right|^{2} \leq\left|\frac{2}{\lambda}\right|^{2} \frac{52}{1+\exp (2(a-b) \ell)+2 \exp ((a-b) \ell) \cos ((a+b) \ell)-128 e^{-b \ell}} \\
& \rightarrow 0 \text { as } n \rightarrow \infty \text { uniformly for all } \lambda \in \Gamma_{n} .
\end{aligned}
$$

Take $N_{1} \geq 0$ such that

$$
\left|\frac{2}{\lambda} \frac{f^{\prime}(\lambda)}{f(\lambda)}\right|^{2}<1 \quad \text { uniformly for all } \lambda \in \Gamma_{n}, \quad n \geq N_{1} .
$$

Applying Rouché's Theorem (see [5]), there is a unique zero point $\hat{\mu}_{n} \in \mathscr{O}_{n}$ for every $n \geq N_{1}$ to the meromorphic function $1+\frac{2 f^{\prime}(\lambda)}{\lambda f(\lambda)}$.

On the other hand, let $\gamma_{1}$ and $\gamma_{2}$ be two lines parallel to $\gamma$ and in contact with every $\mathscr{O}_{n}$ as shown in Figure 2, and let $\mathscr{C}_{n}$ be a closed contour defined as follows. Outside of the region enclosed by the lines $\gamma_{1}$ and $\gamma_{2}, \mathscr{C}_{n}$ is defined by circular arcs of radius $\left(2 \beta_{n}^{2}+\rho^{2}\right)^{\frac{1}{2}}$ centered at the origin. Inside the region enclosed by $\gamma_{1}$ and $\gamma_{2}, \mathscr{C}_{n}$ is defined by the lower section of the $\mathscr{O}_{n}$ nearest to the origin indicated by the thin curve in Figure 2 below. Denote by $\mathscr{D}_{n}$ the region enclosed by $\mathscr{C}_{n}$.

In our case of $b \geq a$, there exists a constant $c_{0}>0$ such that $b-a \geq c_{0}$ for all $(a, b)$ outside of $\mathscr{D}_{n}$ and $\gamma_{1}$, it follows from (19) that there exists an $N_{2} \geq N_{1}$ such that

$$
\left|\frac{2}{\lambda} \frac{f^{\prime}(\lambda)}{f(\lambda)}\right|^{2}<1 \quad \text { outside of } \mathscr{D}_{n} \text { and } \gamma_{1}, \gamma_{2} \text { when } n \geq N_{2}
$$

that is, there is no zero point for $1+\frac{2 f^{\prime}(\lambda)}{\lambda f(\lambda)}$ outside of $\mathscr{D}_{n}$ and the region enclosed by $\gamma_{1}$ and $\gamma_{2}$ when $n \geq N_{2}$. Next, consider the region $\mathscr{G}_{n}$ enclosed by $\mathscr{O}_{n}, \mathscr{O}_{n+1}, \gamma_{1}$ and $\gamma_{2}$. Then

$$
\left|\frac{2}{\lambda} \frac{f^{\prime}(\lambda)}{f(\lambda)}\right|^{2}<1 \quad \text { for } \lambda \in \Gamma \mathscr{G}_{n} \text { and } n \geq N=\max \left\{N_{1}, N_{2}\right\}
$$

and hence there is no zero point for $1+\frac{2 f^{\prime}(\lambda)}{\lambda f(\lambda)}$ in $\mathscr{G}_{n}$ for all $n \geq N$ by Rouché's Theorem. Meanwhile, there are just $N-1$ zero points for $1+\frac{2 f^{\prime}(\lambda)}{\lambda f(\lambda)}$ in $\mathscr{D}_{N}$.

Finally, for $n \geq N$, taking circle $\mathscr{\theta}_{O n}$ to be the circle with its center at $\hat{\lambda}_{n}$ and radius

$$
\alpha_{n}=\frac{1}{\alpha \beta_{n}} \quad \text { for some } \alpha>0,
$$




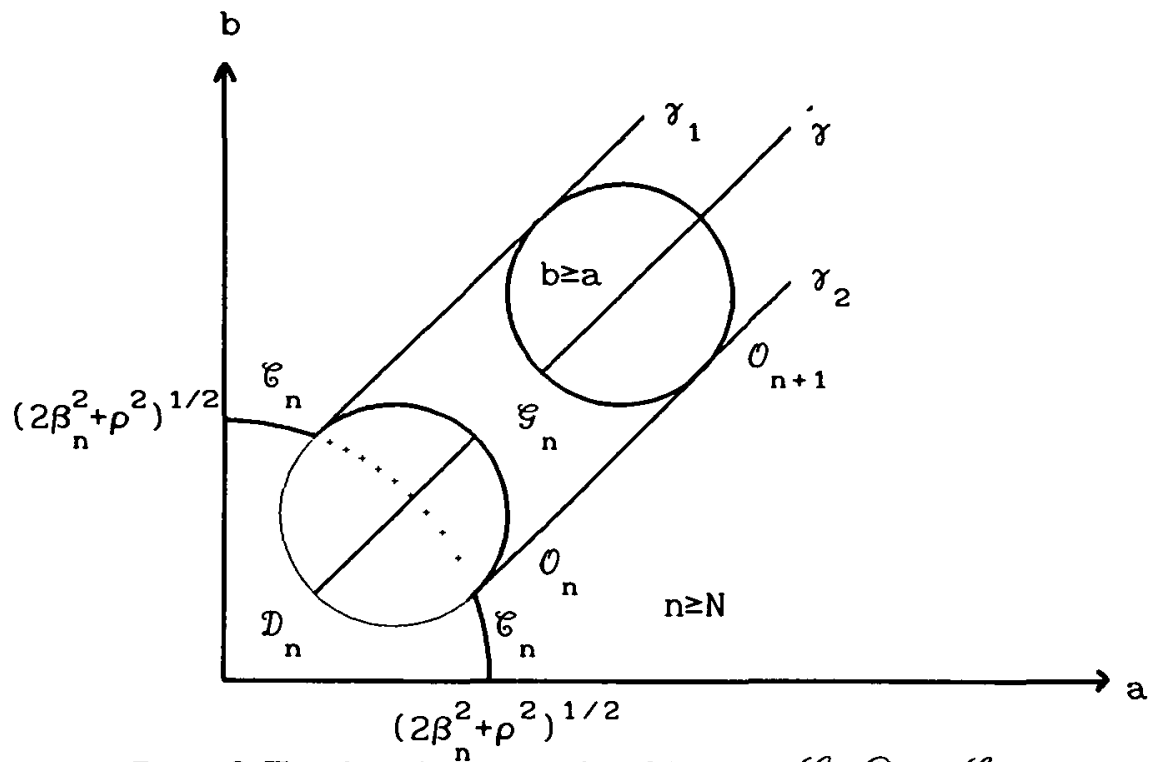

FIGURE 2. The schematic representation of the regions $\mathscr{C}_{n}, \mathscr{D}_{n}$ and $\mathscr{G}_{n}$.

we have

$$
|a-b| \leq \frac{2}{\alpha \beta_{n}}, \quad\left|a+b-2 \beta_{n}\right| \leq \frac{2}{\alpha \beta_{n}}, \quad|\lambda|^{2}=2 \beta_{n}^{2}\left[1+o\left(\beta_{n}^{-1}\right)\right],
$$

where $\lambda=a+b i \in \Gamma_{o_{n}}$, the boundary of $\sigma_{O_{n}}$. From (21)

$$
\begin{aligned}
& 1+\exp (2(a-b) \ell)-2 \exp ((a-b) \ell) \sin ((a+b) \ell) \\
& =1+\exp (2(a-b) \ell)-2 \exp ((a-b) \ell) \sin \left(\left(a+b-2 \beta_{n}+2 \beta_{n}\right) \ell\right) \\
& =1+\exp (2(a-b) \ell)-2 \exp ((a-b) \ell) \sin \left(\left(a+b-2 \beta_{n}\right) \cos \left(2 \beta_{n} \ell\right)\right. \\
& -2 \exp ((a-b) \ell) \cos \left(\left(a+b-2 \beta_{n}\right) \sin \left(2 \beta_{n} \ell\right)+8 e^{-b \ell}\right. \\
& \rightarrow 2 \text { uniformly for all } \lambda \in \Gamma_{\text {on }} \text { as } n \rightarrow \infty,
\end{aligned}
$$

and

$$
\begin{aligned}
& \beta_{n}^{2}[1+\exp (2(a-b) \ell)+2 \exp ((a-b) \ell) \cos ((a+b) \ell)] \\
& =\beta_{n}^{2}[1-\exp (a-b) \ell]^{2}+2 \beta_{n}^{2} \exp ((a-b) \ell)[1+\cos ((a+b) \ell)] \\
& =\beta_{n}^{2}\left[1-\exp \left(\frac{1}{\alpha \beta_{n}}(\cos \theta-\sin \theta) \ell\right)\right]^{2} \\
& +2\left[\exp \left(\frac{1}{\alpha \beta_{n}}(\cos \theta-\sin \theta) \ell\right)\right] \beta_{n}^{2}\left[1-\cos \left(\frac{1}{\alpha \beta_{n}}(\cos \theta+\sin \theta) \ell\right)\right]
\end{aligned}
$$




$$
\begin{aligned}
& +\beta_{n}^{2} o\left(\exp \left(-\beta_{n} \ell\right)\right) \\
& \longrightarrow \alpha^{-2}((\cos \theta-\sin \theta) \ell)^{2}+\alpha^{-2}((\cos \theta+\sin \theta) \ell)^{2}=2 \ell^{2} \alpha^{-2}
\end{aligned}
$$$$
\text { uniformly for all } \lambda \in \Gamma_{O_{n}} \text { as } n \rightarrow \infty \text {. By (20) }
$$

$$
\left|\frac{2}{\lambda} \frac{f^{\prime}(\lambda)}{f(\lambda)}\right|^{2} \rightarrow 2 \ell^{-2} \alpha^{2} \quad \text { uniformly for all } \lambda \in \Gamma_{\text {on }} \text { as } n \rightarrow \infty \text {. }
$$

If

$$
2 \ell^{-2} \alpha^{2} k^{2}<1,
$$

then again by Rouché's Theorem, there exists an $M>N$ such that when $n \geq M$, $\hat{\mu}_{n} \in \mathscr{O}_{O n} \subset \mathscr{O}_{n}$,

$$
\begin{array}{r}
\hat{\mu}_{n}=a_{n}+b_{n} i, \quad a_{n}=\beta_{n}+\frac{1}{\alpha \beta_{n}} \cos \theta, \quad b_{n}=\beta_{n}+\frac{1}{\alpha \beta_{n}} \sin \theta \\
\text { for some } 0 \leq \theta \leq 2 \pi .
\end{array}
$$

If

$$
2 \ell^{-2} \alpha^{2} k^{2}>1
$$

then there is no zero point for $1+k \frac{2 f^{\prime}(\lambda)}{\lambda f(\lambda)}$ in $\mathscr{O}_{O_{n}}$ for $n \geq M>N$. Let

$$
\mu_{n}=\hat{\mu}_{n}^{2} / 2=x_{n}+y_{n} i
$$

Then

$$
\left\{\begin{array}{l}
x_{n}=a_{n}^{2}-b_{n}^{2}=\frac{\sqrt{2}}{\alpha} \cos (\pi / 4+\theta)+\frac{1}{2}\left(\frac{1}{\alpha \beta}\right)^{2} \cos (2 \theta), \\
y_{n}-\beta_{n}^{2}=a_{n} b_{n}=\frac{\sqrt{2}}{\alpha} \sin (\pi / 4+\theta)+\frac{1}{2}\left(\frac{1}{\alpha \beta_{n}}\right)^{2} \sin (2 \theta) \text { for some } 0 \leq \theta \leq 2 \pi,
\end{array}\right.
$$

or

$$
\left|\mu_{n}-i \beta_{n}^{2}\right|=\frac{\sqrt{2}}{\alpha}+o\left(\beta_{n}^{-1}\right) \quad \text { for any } \frac{\sqrt{2}}{\alpha}>2 k / \ell .
$$

Since $2 \ell^{-2} \alpha^{2} k^{2}=1$ if and only if $\frac{\sqrt{2}}{\alpha}=2 k / \ell$, we obtain the required result by choosing $\mathscr{O}_{n}$ to be the circle with center at $\sqrt{\lambda_{n}} i$ and radius $2 k / \ell$.

Theorem 2 indicates that the system operator $\mathscr{A}$ defined in Theorem 1 cannot have an analytic extension. The fact that the decay rate $-\omega$ in Theorem 1 is not proportional to the feedback constant $k$ is established in the following theorem. 
THEOREM 3. The set of zero points of the entire function $f^{\prime}(\sqrt{2 \lambda})$ defined by (12) consists of purely complex numbers $\left\{ \pm i \alpha_{n}^{2}\right\}$ which can be obtained from

$$
\tan \left(\alpha_{n} \ell\right)=2 /\left(1+e^{2 \alpha \ell}\right)-1
$$

and

$$
\alpha_{n}=o(n), \quad 0<\alpha_{1}<\beta_{1}, \quad \beta_{n-1}<\alpha_{n}<\beta_{n} \text { for } n \geq 1 .
$$

For any given $N>0$, let $\left\{\mu_{n}, \bar{\mu}_{n}\right\}$ be the eigenvalues of (5). Then

(i) $\max _{1 \leq n \leq \infty}\left\{\left|\mu_{n}-i \beta_{n}^{2}\right|,\left|\bar{\mu}_{n}+i \beta_{n}^{2}\right|\right\} \rightarrow 0$ as $k \rightarrow 0$;

(ii) $\max _{1 \leq n \leq N}\left\{\left|\mu_{n}-i \alpha_{n}^{2}\right|,\left|\bar{\mu}_{n}+i \alpha_{n}^{2}\right|\right\} \rightarrow 0$ as $k \rightarrow \infty$.

PROOF. We only consider (ii), as the proof of (i) is similar. Let $\sqrt{2 \lambda_{0}}=a+b i$ be a zero point of $f^{\prime}(\sqrt{2 \lambda})$. Then

$$
\begin{aligned}
& \left(e^{-a \ell}-e^{a \ell}\right) \cos (b \ell)+\left(e^{b \ell}+e^{-b \ell}\right) \sin (a \ell)=0, \\
& \left(e^{-b \ell}-e^{b \ell}\right) \cos (a \ell)+\left(e^{a \ell}+e^{-a \ell}\right) \sin (b \ell)=0,
\end{aligned}
$$

which is symmetric about $a$ and $b$. Since $2 \lambda_{0}=a^{2}-b^{2}+2 a b, 2 \lambda_{1}=b^{2}-a^{2}+2 a b$ is still a zero point of $f^{\prime}(\sqrt{2 \lambda})$. If $a \neq b$, then either $\operatorname{Re} \lambda_{0}>0$ or $\operatorname{Re} \lambda_{1}>0$. Suppose $\lambda_{0}<0$. Then consider a circle $\mathscr{O}$ located in the right half complex plane with center at $\lambda_{0}$ so that $\lambda_{0}$ is the only zero point of $f^{\prime}(\sqrt{2 \lambda})$ inside $\mathscr{O}$ and $\Gamma \mathscr{O}$. Let $k>0$ be large enough so that

$$
|f(\sqrt{2 \lambda})|<k / \ell\left|f^{\prime}(\sqrt{2 \lambda})\right| \quad \text { on } \Gamma \mathscr{O} .
$$

By Rouché's theorem, there is one zero point of $f(\sqrt{2 \lambda})+k / \ell f^{\prime}(\sqrt{2 \lambda})$ inside $\mathscr{O}$ which contradicts Lemma 2 and Lemma 3 . So $\lambda_{0}= \pm \alpha^{2} i$ is a purely complex number and $\alpha$ satisfies (29) and (30).

Next, for every $-N \leq n \leq N$, take $\mathscr{O}_{n}$ to be the cycle with center at $i \alpha_{n}^{2}$ such that $i \alpha_{n}^{2}$ is the only zero point of $f^{\prime}(\sqrt{2 \lambda})$ inside $\mathscr{O}_{n}$ and $\Gamma \mathscr{O}_{n}$. Let $k>0$ be large enough so that

$$
\ell|f(\sqrt{2 \lambda})| / k<\left|f^{\prime}(\sqrt{2 \lambda})\right| \quad \text { for all } \Gamma \mathscr{O}_{n},-N \leq n \leq N .
$$

Then, again by Rouché's Theorem, there is a unique zero point inside $\mathscr{O}_{n}$ to the entire function $\ell f(\sqrt{2 \lambda}) / k+f^{\prime}(\sqrt{2 \lambda})$. Part (ii) is obtained by Lemma 3 .

\section{Acknowledgement}

The author would like to thank Dr Z. H. Luo for giving him his paper [3] and for several helpful discussions, which, in turn, motivated him to consider the problem of this paper. 


\section{References}

[1] G. Chen, M. C. Delfour, A. M. Krall and G. Payre, "Modeling, stabilization and control of serially connected beams", SIAM J. Control \& Optimization 25 (1987) 526-546.

[2] H. G. Lee, F. Miyazaki and S. Arimoto, "Liapunov stability analysis for PDS control of flexible multilink manipulator", Proc. of 27th IEEE on CDC 1 (1988) 75-80.

[3] Z. H. Luo, "Direct strain feedback control of flexible arms: new theoretical and experimental results", IEEE Trans. on Automatic Control 38 (1993) 1610-1622.

[4] Z. H. Luo and B. Z. Guo, "Further theoretical results on direct strain feedback control of flexible robot arms", IEEE Trans. on Automatic Control 40 (1995).

[5] E. C. Titchmarsh, The theory of functions (Oxford University Press, 1952) (corrected). 